\title{
MAPS PRESERVING THE LOCAL SPECTRUM OF SOME MATRIX PRODUCTS
}

\section{Zine El Abidine AbDelali, AbDelali AchCHi And Rabi Marzouki}

Abstract. Let $\mathscr{M}_{n}(\mathbb{C})$ denote the algebra of all $n \times n$ complex matrices, and $x_{0}$ a nonzero vector in $\mathbb{C}^{n}$. For two fixed scalars $\mu$ and $v$ in $\mathbb{C}$ for which $(\mu, v) \neq(0,0)$, we characterize all maps $\varphi$ on $\mathscr{M}_{n}(\mathbb{C})$ satisfying

$$
\sigma_{\mu S T^{*} S+v T^{*} S}\left(x_{0}\right)=\sigma_{\mu \varphi(S) \varphi(T)^{*} \varphi(S)+v \varphi(T)^{*} \varphi(S)}\left(x_{0}\right), \quad\left(S, T \in \mathscr{M}_{n}(\mathbb{C})\right) .
$$

This provides, in particular, a complete description of all maps on $\mathscr{M}_{n}(\mathbb{C})$ preserving the local spectrum of the skew double product " $T S^{*}$ " or the skew triple product " $T S^{*} T$ " of matrices. It also unifies and extends several known results on local spectrum preservers.

Mathematics subject classification (2010): Primary 47B49, Secondary 47A10, 47A11.

Keywords and phrases: Nonlinear preservers, local spectra, skew double product, skew triple product.

\section{REFERENCES}

[1] Z. AbDelali, A. ACHCHI And R. MaRzouKi, Maps preserving the local spectrum of skew-product of operators, Linear Algebra Appl. 485 (2015), 58-71.

[2] P. AIEnA, Fredholm and Local Spectral Theory, with Applications to Multipliers, Kluwer Academic Publishers, Dordrecht, 2004.

[3] G. An And J. Hou, Rank-preserving multiplicative maps on $\mathscr{B}(X)$, Linear Algebra Appl. 342 (2002), 59-78.

[4] B. Aupetit, A Primer on Spectral Theory, Universitext, Springer-Verlag, New York, 1991.

[5] M. Bendaoud, Preservers of local spectrum of matrix Jordan triple products, Linear Algebra Appl. 471 (2015), 604-614.

[6] M. BendaOUd, M. JABBAR AND M. SARIH, Preservers of local spectra of operator products, Linear Multilinear Algebra 63 (4) (2015), 806-819.

[7] R. BhAtiA, P. ŠEMrL AND A. SOURour, Maps on matrices that preserve the spectral radius distance, Studia Math. 134 (2) (1999), 99-110.

[8] A. Bourhim And M. Mabrouk, Jordan product and local spectrum preservers, Studia Math. 234 (2) (2016), 97-120.

[9] A. BOURHIM AND M. MABROUK, Maps preserving the local spectrum of Jordan product of matrices, Linear Algebra Appl. 484 (2015), 379-395.

[10] A. Bourhim AND J. MAShreGHI, Local spectral radius preservers, Integral Equations Operator Theory 76 (1) (2013), 95-04.

[11] A. Bourhim AND J. MASHREGHI, Maps preserving the local spectrum of product of operators, Glasgow Math. J. 57 (3) (2015), 709-718.

[12] A. BOURHIM AND J. MASHREGHI, Maps preserving the local spectrum of triple product of operators, Linear Multilinear Algebra 63 (4) (2015), 765-773.

[13] A. Bourhim AND J. MASHREGHI, A survey on preservers of spectra and local spectra, in: Invariant Subspaces of the Shift operator, Contemp. Math. 638, Amer. Math. Soc, Providence, RI (2015), 45-98.

[14] A. Bourhim And V. G. Miller, Linear maps on $\mathscr{M}_{n}(\mathbb{C})$ preserving the local spectral radius, Studia Math. 188 (1) (2008), 67-75.

[15] A. BOURHIM AND T. RANSFORD, Additive maps preserving local spectrum, Integral Equations Operator Theory 55 (2006), 377-385. 
[16] J. T. Chan, C. K. Li AND N. S. SZE, Mappings preserving spectra of products of matrices, Proc. Amer. Math. Soc. 135 (2007), 977-986.

[17] C. CostaRA, Linear maps preserving operators of local spectral radius zero, Integral Equations Operator Theory 73 (1) (2012), 7-16.

[18] J. L. CUI AND J. C. Hou, Maps leaving functional values of operator products invariant, Linear Algebra Appl. 428 (2008), 1649-1663.

[19] J. L. CUI AND C. K. LI, Maps preserving peripheral spectrum of Jordan products of operators, Oper. Matrices 6 (2012), 129-146.

[20] M. Dollinger And K. Oberai, Variation of local spectra, J. Math. Anal. Appl. 39 (1972), 324 337.

[21] M. GonZÁLeZ And M. MbeKhta, Linear maps on $\mathscr{M}_{n}(\mathbb{C})$ preserving the local spectrum, Linear Algebra Appl. 427 (2007), 176-182.

[22] J. C. Hou AND Q. H. Di, Maps preserving numerical range of operator products, Proc. Amer. Math. Soc. 134 (2006), 1435-1446.

[23] M. Marcus AND B. N. Moyls, Linear transformations on algebras of matrices, Canad. J. Math. 11 (1959), 61-66.

[24] T. MiURA AND D. Honma, A generalization of peripherally-multiplicative surjections between standard operator algebras, Cent. Eur. J. Math. 7 (3) (2009), 479-486.

[25] L. Molnár, Some characterizations of the automorphisms of $B(H)$ and $C(X)$, Proc. Amer. Math. Soc. 130 (1) (2002), 111-120.

[26] K. B. Laursen And M. M. Neumann, An Introduction to Local Spectral Theory, London Math. Soc. Monographs (N.S.) 20, Calderon Press, Oxford, 2000.

[27] C. K. LI, P. ŠEMRL AND N. S. SZE, Maps preserving the nilpotency of products of operators, Linear Algebra Appl. 424 (2007), 222-239.

[28] R. A. Horn And C. R. Johnson, Matrix Analysis, 2nd edition, Cambridge University Press, Cambridge, 2012.

[29] M. WANG, L. FANG AND G. Ji, Linear maps preserving idempotency of products or triple Jordan products of operators, Linear Algebra Appl. 429 (2008), 181-189.

[30] W. Zhang AND J. Hou, Maps preserving peripheral spectrum of Jordan semi-triple products of operators, Linear Algebra Appl. 435 (2011), 1326-1335. 